\title{
NETWORK PERFORMANCE OPTIMIZATION
}

\author{
David Grenar \\ Doctoral Degree Programme (3.), FEEC BUT \\ E-mail: xgrena04@vutbr.cz \\ Supervised by: Miloslav Filka \\ E-mail: filka@feec.vutbr.cz
}

\begin{abstract}
It is necessary to monitor the usage of network resources within the network, and provide the solution in order to avoid network congestion problems. Based on it, a real-time network traffic analysis followed and optimization of the performance benefits. Paper will implement solution of congestion problems with packets, which are processed in RouterOS. This method will combine with mangle, firewall rules, so that we can manage the internet connection to our network ideally, and prioritize the connection packet that we want to get priority in size as we need.
\end{abstract}

Keywords: Mikrotik RouterOS, Performance, Implementation, Congestion

\section{INTRODUCTION}

Network is infrastructure of each application system, usually consists of multiple switches, routers, and security devices. It is necessary to monitor the usage of network resources within the network, and give the solution to avoid network congestion problems. With the changing communication requirements, the data flow through WAN shows unpredictable, uncontrollable trend. On the other hand, the transmission channel's instability and easy to be interrupted. Traffic in network cannot be controlled very well. In order to smooth communication process, timely detection and making resolution of traffic within the uncontrollable hidden dangers, there is an urgent need for real-time analysis of traffic and congestion management [1].

Multimedia applications require the transmission of real-time streams over a network. These streams often exhibit variable requirements, and require high bandwidths and guarantees from the network. This creates problems when such streams are delivered over the Internet.

Manage the internet connections from the ISP to the local network for some kind of internet purposes effectively. I hope this can be one of the reference for you to manage the internet connection as you intended. Of course you have to understand your internet network environment, that you have, so that you can implemented as you needed [2].

\section{ROUTEROS}

Mikrotik RouterOS is an operating system and software that can be used in order to make the computer a reliable network router, covering various features including: firewall and NAT, routing, hotspot, point to point tunneling protocol, DNS server, DHCP server, hotspot, burst and still many more features and functions. Mikrotik is suitable for use by ISP. For the installation of Mikrotik is not required additional software or other additional components. Mikrotik is designed to be easy to use and very well used for the purposes of computer network administration, such as designing and building a small complex computer network system though. Based on the form of hardware in use, Mikrotik can be classified into two types [3]:

- Mikrotik RouterOS in the form of ISO software from RouterOS which can be downloaded at www.mikrotik.com. 
- Mikrotik Routerboard (Embedded Router) in the form of hardware that is specifically packed include with Mikrotik RouterOS.

In network management, it is very important to do usage management that will be used by each user. Mikrotik routers have queue function and facilities that can perform bandwidth allocation management for each user's computer. By implementing bandwidth management, is aim to improve the quality of service on the network (Quality of Service). Quality of Service (QoS) will guarantee the minimum bandwidth allocation / Committed Information Rate (CIR) on each user's computer in the network and the bandwidth allocation over Maximum Information Rate (MIR) within a certain time period, so each computer user will not have to worry about not get the bandwidth $[4,5]$.

Firewall in Mikrotik have main functions to protect network from wan network accident and take care about sensitive date in our network. Whenever we have different networks and they are connected together, there are always important to use necessarily protection rules which are important in order to protect our communications infrastructure. Properly configured firewall plays a key role in efficient and secure network infrastructure deployment [5].

\subsection{SLOW PATH}

Slow Path is the regular way packets and it is processed in RouterOS. For each packet, RouterOS has to check the whole path of the packet. In some cases, it is a considerable number of steps, which can affects performance. Slow Path consumes more resources than Fast Path [6].

\subsection{FAST PATH}

Fast Path is a feature of the Linux kernel and RouterOS uses the Linux kernel. Fast Path is an interface driver extension that allows you to receive, process or send traffic without unnecessary processing. Interface driver can now talk directly to specific RouterOS processes - skipping all others steps. You can use Fast Path in order to achieve compatibility within Slow Path devices. Fast Path could be from RouterOS 6.30 used on logical interfaces such a Bridge, VLAN, VRRP and Bonding - receive only. Since RouterOS 6.33 for EOIP, GRE, IPIP and since 6.36 PPPoE client interface. Traffic traveling in Fast Path will be invisible to other router facilities (firewall, queues, etc). This is show on Figure 1. [5, 6, 8]

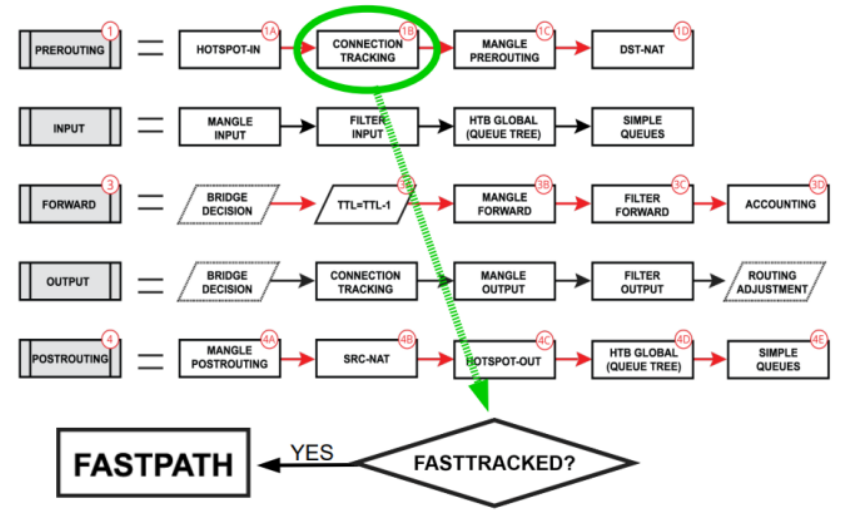

Obrázek 1: $\quad$ Diagram for routing forwarding by Fast Path. [6]

\subsection{FAST TRACK}

MikroTik integrates Fast Path in previous version in ROS 6.30. Fast Path don't include Fast Path and Conntrack. In new RouterOS 6.42 was added new flag fasttracked. This function was integrated to firewall filter and mangle. Fasttracked packet connections also use Fast Path. Currently only TCP and UDP connections can be actually Fast Tracked (even though any connection can be marked for FastTrack). IPv4 FastTrack handler supports NAT (SNAT, DNAT or both). [7, 8] 


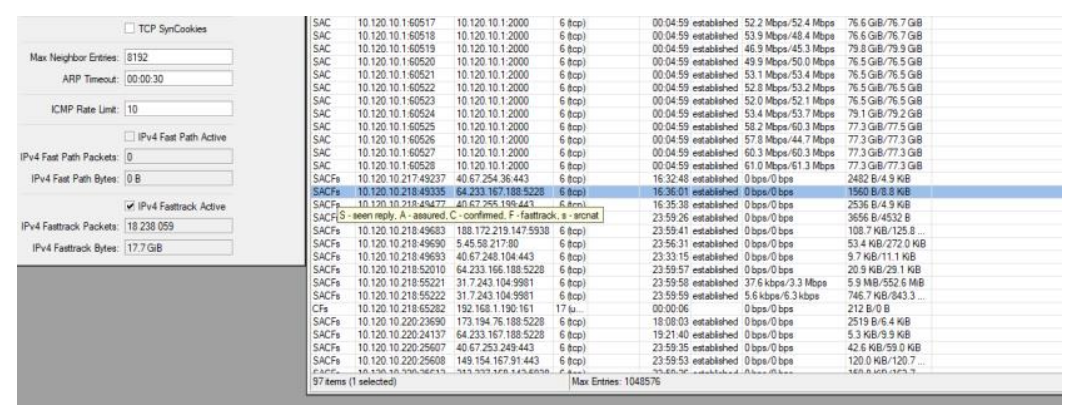

Obrázek 2: $\quad$ Fasttracked connection in firewall.

All packets in a connection can be Fast Tracked, so it is likely to see some packets going through slow path. Even though connection is marked for FastTrack. This is the reason, why fasttrackconnection is usually followed by identical action=accept rule. Fast Tracked packets bypass firewall, connection tracking, simple queues, queue tree with parent=global, ip traffic-flow (restriction removed in 6.33), IP accounting, IPSec, hotspot universal client, VRF assignment, so it is up to administrator to make sure, that FastTrack does not interfere with other configuration [9];

Requirements for FastTrack function:

- no mesh, metarouter interface configuration;

- sniffer, torch and traffic generator is not running;

- /tool mac-scan is not actively used;

- /tool ip-scan is not actively used.

\subsection{IMPLEMENTATION}

Fast Track accelerates the processing of packets, because it started working on firmware from RouterOS 6.29. The feature rules within router firewall filter can be set-up depending on individual needs. It will enable for Fasttracked packets bypass firewall of typical connection packets, that are intended. To activate Fast Track is necessary to add to ip firewall filter rules with action fasttrackconnection $[6,10]$.

Setting example for Fast Track in terminal by command:

/ip firewall filter add chain=forward action=fasttrack-connection connection-state=established,related

/ip firewall filter add chain=forward action=accept connection-state=established,related

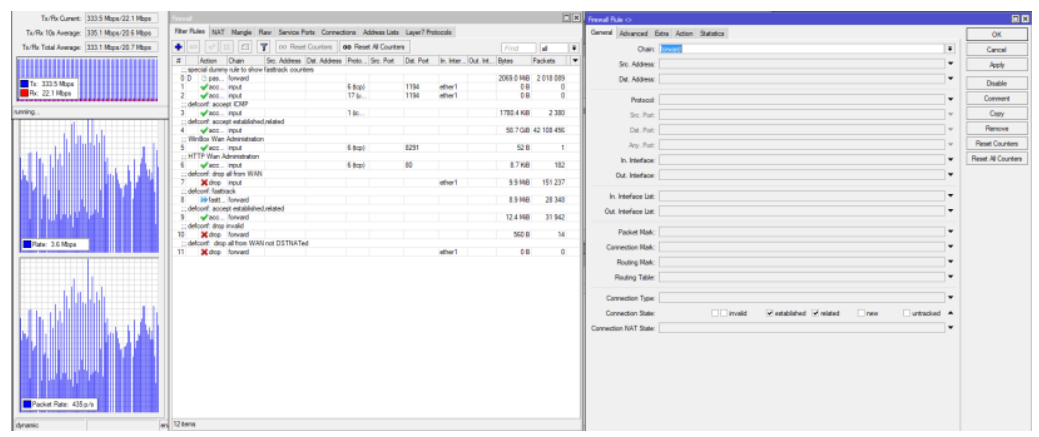

Obrázek 3: Monitoring performance after application of Fast Track. 


\subsection{PeRFormanCE}

In our testing we have Mikrotik RB3011UiAS-RM with RouterOS 6.44. Traffic, which was used, has CPU capacity on almost full CPU source. In our default setup, our connections were processed with Fast Path function. That's why we trying to found out a way to save CPU usage and optimization performance within our network. The throughput of this network was another parameter which was change by Fast Track as the pass through. At the figure 4 is on left graph captured CPU using without Fast track and on the right is Graf routing with Fast track. By using this configuration, we are allowed to reduce CPU load $[6,9]$.
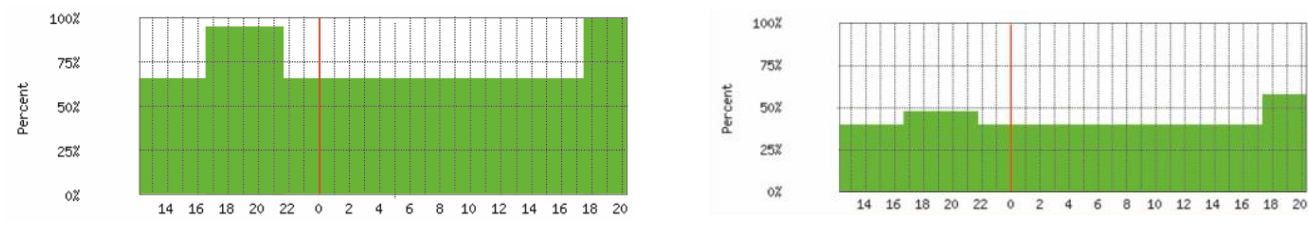

Obrázek 4: Modification load of CPU usage by Fast Track functions.

Main part of reduction of load at router was also higher efficiency of the throughput and better load of CPU using IP/Firewall profile which works with CPU. On table 1, we have compared single TCP test with Fast Path and Fast Track. From this table we are able to see that Fast Track raping the throughput and decreases the load of CPU. This performance optimization has effect for shaping and also detection of specific type of contend connections services, for example like video streaming. RouterOS uses receive flow packet steering to assign incoming traffic to a specific CPU thread, which is hash value. Hashing process can be hard-coded in the hardware, configurable in the hardware or implemented in the interface driver. This futures flow steering will try to keep single TCP stream bound to single CPU thread as long as possible. These properties have impact on RTT (round-trip time), because this save processing time and reduce packet loss. [11, 13].

\begin{tabular}{|l|l|l|l|}
\hline & $\begin{array}{l}\text { Traffic throughput } \\
\text { (TCP) }\end{array}$ & $\begin{array}{l}\text { Load of } \\
\text { CPU }\end{array}$ & $\begin{array}{l}\text { Profile CPU } \\
\text { (Firewall) }\end{array}$ \\
\hline Fast Path & $353 \mathrm{Mb} / \mathrm{s}$ & $97 \%$ & $38 \%$ \\
\hline Fast Track & $835 \mathrm{Mb} / \mathrm{s}$ & $53 \%$ & $8 \%$ \\
\hline
\end{tabular}

Tabulka 1: $\quad$ Change throughput and CPU by Fast Track.

\section{CONCLUSION}

Fasttrack-connection filter rule looks similar to mark-connection action in configuration. This rule is mostly followed by identical accept rule. Fast track is used if connection is established and related. Common of Fast track is to exclude some specific connections from the queues. From this information we can considerate that FastTrack accelerated steps in our connections and save CPU and bring better performance for throughput in our network. The feature rules in router firewall filter that you can setup depending on your individual needs. It will Fasttracked packets bypass firewall of typical connection packets that you intended. Properly implement both of these rules it work correctly, and reaping the performance benefits.

\section{ACKNOWLEDGEMENT}

The presented research has been supported by the internal BUT project FEKT-S-17-4184. 


\section{REFERENCES}

[1] HART, Tyler. Networking with MikroTik: MTCNA Study Guide. 2017. ISBN 9781973206354.

[2] DISCHER, Stephen, RouterOS by Example, 2nd Edition: B\&W: B\&W Version. ISBN 9780692777084.

[3] MikroTik RouterOS: RouterOS software documentation. MikroTik Wiki: MikroTik documentation wiki [online]. Available from: https://wiki.mikrotik.com/wiki/Main_Page

[4] LESMANA SIAHAAN, Muhammad Donni, Melva SARI PANJAITAN a Andysah Putera UTAMA SIAHAAN. MikroTik Bandwidth Management to Gain the Users Prosperity Prevalent. In: International Journal of Engineering Trends and Technology. 2016, 42(5), s. 218222. DOI: 10.14445/22315381/IJETT-V42P243. ISSN 22315381

[5] MikroTik RouterOS: Routing Forwarding [online]. In: 2015. Available from: https://mum.mikrotik.com/presentations/UA15/presentation_3077_1449654925.pdf

[6] TURKAN, Himmet. MUM: ISP Network Design. In: Mum.mikrotik.com [online]. Türkiye: MUM, 2018. Available from: https://mum.mikrotik.com/presentations/TR18/

[7] FURINI, M. a D.F. TOWSLEY. Real-time traffic transmission over the Internet. In: IEEE Transactions on Multimedia [online]. 3(1), s. 33-40. DOI: 10.1109/6046.909592. ISSN 15209210. Available from: http://ieeexplore.ieee.org/document/909592/

[8] O'HALLORAN, Cian. Dynamic adaptation of OSPF interface metrics based on network load. In: 2015 26th Irish Signals and Systems Conference (ISSC). IEEE, 2015, 2015, s. 1-6. DOI: 10.1109/ISSC.2015.7163767. ISBN 978-1-4673-6974-9. Available from: http://ieeexplore.ieee.org/document/7163767/

[9] YAMAMOTO, Shu a Akihiro NAKAO. Fast Path Performance of Packet Cache Router Using Multi-core Network Processor. In: 2011 ACM/IEEE Seventh Symposium on Architectures for Networking and Communications Systems. IEEE, 2011, 2011, s. 89-90. DOI: 10.1109/ANCS.2011.22. ISBN 978-1-4577-1454-2. Available from: http://ieeexplore.ieee.org/document/6062718/

[10] CUEVA, H., F. POZO a D. ITURRALDE. Cross-platform network visualization software for MikroTik devices. In: 2016 IEEE ANDESCON. IEEE, 2016, 2016, s. 1-4. DOI: 10.1109/ANDESCON.2016.7836222. ISBN 978-1-5090-2532-9. Available from: http://ieeexplore.ieee.org/document/7836222/

[11] HUNDESSA, L. a J.D. PASCUAL. Fast rerouting mechanism for a protected label switched path. In: Proceedings Tenth International Conference on Computer Communications and Networks (Cat. No.01EX495). IEEE, 2001, s. 527-530. DOI: 10.1109/ICCCN.2001.956316. ISBN 0-7803-7128-3. Available from: http://ieeexplore.ieee.org/document/956316/

[12] PAUZHI, W. a J. CORONEL. Security for WISP through Mikrotik equipment. In: 2015 CHILEAN Conference on Electrical, Electronics Engineering, Information and Communication Technologies (CHILECON). IEEE, 2015, 2015, s. 229-233. DOI: 10.1109/Chilecon.2015.7400381. ISBN 978-1-4673-8756-9. Available from: http://ieeexplore.ieee.org/document/7400381/

[13] LiU, Z., J. ALMHANA, V. CHOUlaKiAN a R. MCGORMAN. Periodic Data Traffic Modeling and Predicition-Based Bandwith Allocation. In: 4th Annual Communication Networks and Services Research Conference (CNSR'06). IEEE, 2006, s. 131-138. DOI: 10.1109/CNSR.2006.41. ISBN 0-7695-2578-4. Available from: http://ieeexplore.ieee.org/document/1628108/ 